\title{
Generation and characterization of bioluminescent xenograft mouse models of MLL-related acute leukemias and in vivo evaluation of luciferase-targeting siRNA nanoparticles
}

\author{
RAFFAELLA FAZZINA ${ }^{1}$, LORENZA LOMBARDINI ${ }^{1}$, LAURA MEZZANOTTE ${ }^{2}$, \\ ALDO RODA $^{2}$, PATRIZIA HRELIA ${ }^{3}$, ANDREA PESSION $^{1}$ and ROBERTO TONELLI ${ }^{3}$ \\ ${ }^{1}$ Department of Pediatric Oncology and Hematology, Sant' Orsola Hospital, University of Bologna, Bologna; \\ Departments of ${ }^{2}$ Pharmaceutical Sciences and ${ }^{3}$ Pharmacology, University of Bologna, Bologna, Italy
}

Received February 2, 2012; Accepted April 13, 2012

DOI: 10.3892/ijo.2012.1504

\begin{abstract}
Chromosomal translocations involving the MLL gene on 11q23 present frequent abnormalities in pediatric, adult and therapy-related acute leukemias, and are generally associated with aggressive disease and poor prognosis. Here, we report bioluminescent acute leukemia xenograft mouse models of the most frequent and aggressive MLL-related acute leukemias (infant and adult MLL-AF9, MLL-ENL, MLL-AF4). Four acute leukemia cell lines carrying MLL-related translocations were stably transduced with a firefly luciferase transgene and injected intravenously into NOD/SCID mice. Leukemia progression was monitored by in vivo bioluminescence imaging (BLI). All mice developed MLL-related acute leukemia. The four MLL-related acute leukemia models showed a different course of infant and adult MLL-AF9 acute myeloid leukemia, and a rapid aggressiveness of MLL-ENL acute lymphoblastic leukemia and MLL-AF4 acute biphenotypic leukemia. Tissue analysis and RT-PCR of bone marrow, spleen and liver from the mice confirmed the BL results. To validate BLI for the detection of a therapeutic response, systemic treatment with an anti-luciferase-targeting siRNA (siLuc) complexed with cationic nanoparticles was administered to mice with MLL-AF4 acute lymphoblastic leukemia. The BLI signal showed a reduction following treatment with siLuc compared to the control mice. These mouse models present MLL-related acute leukemia evolution similar to the human counterparts. Moreover, they are non-invasive, rapid and sensitive models, suitable for the in vivo study of
\end{abstract}

Correspondence to: Professor Roberto Tonelli, Department of Pharmacology, University of Bologna, Via Irnerio 48, I-40126 Bologna, Italy

E-mail: roberto.tonelli@unibo.it

Key words: in vivo bioluminescence imaging (BLI), MLL-related acute leukemia, xenograft mouse model, luciferase-targeting siRNA, nanoparticle complex, drug delivery, biotechnologic drug development
MLL-related acute leukemias. Finally, BLI showed in vivo luminescence downmodulation obtained by systemic treatment with luciferase-targeting siRNA nanoparticle complexes, confirming that these MLL-related leukemia mouse models are optimal for the evaluation and selection of delivery systems for siRNA and other new biotechnological pharmaceuticals.

\section{Introduction}

The mixed-lineage leukemia (or myeloid-lymphoid leukemia, MLL) gene on chromosome 11q23 is a common target for chromosomal translocation associated with pediatric, adult and therapy-related acute leukemias, and are generally associated with aggressive disease and poor prognosis (1-4).

Acute lymphoblastic leukemia (ALL) carrying 11q23/MLL translocations represents $8-10 \%$ of total ALL and $60-70 \%$ of infant (i.e. $<1$ year old) ALL $(5,6)$. The most common fusion rearrangement in ALL is the $\mathrm{t}(4 ; 11)(\mathrm{q} 21 ; \mathrm{q} 23)$ MLL-AF4 translocation, representing $5-10 \%$ of adult ALL, up to $5 \%$ of pediatric ALL and $>60 \%$ of infant ALL. The t(4;11)(q21;q23) MLL-AF4 translocation is associated with high rates of early treatment failure $(7,8)$. Leukemic cells carrying the $t(4 ; 11)$ (q21;q23) MLL-AF4 translocation may manifest some cytochemical and ultrastructural features of monocytes, and thus $\mathrm{t}(4 ; 11)(\mathrm{q} 21 ; \mathrm{q} 23)$ MLL-AF4 leukemia has been classified as biphenotypic leukemia (9). The $\mathrm{t}(11 ; 19)(\mathrm{q} 23 ; \mathrm{p} 13.3)$ MLL-ENL translocation represents $10 \%$ of the 11q23 cases in ALL and 5\% in AML M4/M5 (8).

Acute myeloid leukemia (AML) with rearrangements of 11q23/MLL occurs in 10-15\% of children (1-18 years old), $60-70 \%$ of infants and $8-10 \%$ of adult patients $(6,10)$. The $\mathrm{t}(9 ; 11)(\mathrm{p} 22 ; \mathrm{q} 23)$ MLL-AF9 translocation is the most frequent rearrangement in AML subtype M5a, that represents approximately $15 \%$ of AML in children $>2$ years old and approximately $50 \%$ of AML in younger children (8). $\mathrm{t}(9 ; 11)$ (p22;q23) MLL-AF9 leukemia has a more favorable prognosis than $\mathrm{t}(4 ; 11)(\mathrm{q} 21 ; \mathrm{q} 23)$ MLL-AF4 leukemia (11), but the event-free and overall survival rates in AMLs vary largely depending on the MLL fusion partner gene involved, with $\mathrm{t}(9 ; 11)(\mathrm{p} 22 ; \mathrm{q} 23)$ MLL-AF9 having the most favorable outcome and $\mathrm{t}(6 ; 11)(\mathrm{q} 27 ; \mathrm{q} 23)$ MLL-AF6 the poorest (3). 
In vivo bioluminescence imaging (BLI) has become a powerful tool to evaluate cellular and molecular physiologic and pathologic processes in living systems (12-15). BLI has been used successfully in small animal models to study several different types of tumor, such as prostate, breast, colon, ovarian as well as lung cancer and is particularly appropriate for haematopoietic tumors (16-22). BLI is based on the activity of the enzyme luciferase, produced by firefly Photinus pyralis, that acts as a bioluminescent reporter gene. Luciferase catalyzes the oxidation of its substrate, D-luciferin, in the presence of ATP, molecular oxygen and $\mathrm{Mg}^{2+}$, conditions available only in viable cells, with emission of light proportional to the expression of the enzyme luciferase. Expression of luciferase may be accomplished in cells through stable transfection of luciferase under the control of a constitutive promoter (23). For these features, BLI is cost-effective, does not need the use of radioisotopes, is simple, sensitive and provides a strong light signal with virtually no background noise because of the emission of green light from firefly luciferase/luciferin reaction at approximately $612 \mathrm{~nm}$ at body temperature. For in vivo BLI, experimental animals, that do not physiologically express luciferase, are first inoculated with cells that stably express luciferase, then the substrate D-luciferin is intraperitoneally (i.p.) administered to the animals, and light emission from the luciferase-expressing cells is detected with an ultrasensitive charge-coupled device (CCD) camera (24). The results can be presented as qualitative pseudocolor images, representing the number of photons emitted per area, or as quantitative photon counts. It is also possible to establish a correlation between luminescence intensity and cell expansion, since light emission increases as the luciferase-expressing cells multiply in a linear relationship (14). In vivo BLI is a rapid and non-invasive method that permits whole-body studies of experimental animals, preserving at the same time the wellness of the animals, and that can be performed repeatedly to monitor the leukemia growth kinetics and therapeutic efficacy using each animal as its own control. Moreover, the high sensitivity of BLI enables the early detection of very small amounts of luciferase-expressing cells and of deep tumor, preceding the appearance of evident symptoms and blood dissemination (25).

Small interfering RNAs (siRNAs) are double stranded RNAs that mediate RNA interference (RNAi), an evolutionarily conserved sequence-specific post-transcriptional gene silencing (PTGS) mechanism, that consists of the degradation of complementary mRNA or of the inhibition of mRNA translation $(26,27)$. siRNAs are derived from long double stranded RNA (dsRNA) precursors, processed by RNase-III-like enzyme Dicer in 21 nucleotide siRNAs. siRNAs are subsequently rearranged into the RNA induced silencing complex (RISC) that mediates mRNA-target degradation. Alternatively, RNAi can be mediated by synthetic siRNAs, introduced in the cell by delivery methods such as electroporation, cationic liposomes, and nanoparticles. Preferred RNAi targets are viral genes, oncogenes or aberrant gain-of-function genes. RNAi has become the method of choice to suppress gene expression in vitro and it is also emerging as a powerful tool for in vivo research (28-31).

The success of the clinical use of oligonucleotide therapeutics is subdued by their in vivo delivery systems, that should overcome physiological barriers, such as degradation by nucleases in serum and tissue, rapid excretion by glomerular filtration and renal system, phagocytosis and then degradation by circulating macrophages of the reticuloendothelial system, difficulty to cross the capillary endothelial cells, trapping and slow diffusion through the extracellular matrix. The challenge in vivo is constituted by the evaluation and selection of optimal delivery systems for siRNAs and other oligonucleotides to overcome these barriers.

In this respect, several methods have been evaluated: chemically modified oligonucleotides and different delivery systems that can be complexed with siRNA or other DNA-based oligonucleotides, such as cationic liposomes, nanoparticles, cell penetrating peptides (CPP). Cationic nanoparticles are interesting because of their proton sponge effect: they fuse with the cellular membrane, facilitate endocellular release of siRNAs, act as a buffer for the acid $\mathrm{pH}$ of lisosomes and avoid lisosome fusion and siRNA pH-dependent destruction. However, the optimization of the in vivo delivery systems remains critical for a broad clinical success of therapeutic gene targeting by oligonucleotides.

Here we report bioluminescent acute leukemia xenograft mouse models of the most frequent and high risk MLL-related acute leukemias (infant and adult MLL-AF9, MLL-ENL, MLL-AF4). We transduced MLL-related cell lines with firefly luciferase-expressing plasmid, then we inoculated luciferase-expressing cells in NOD/SCID mice to generate in vivo bioluminescent xenograft mouse models of leukemias. The intensity of the BLI signals was investigated to explore the correlation with the progression and distribution of leukemia in the animal models for in vivo study of MLL-related leukemias. To validate BLI for the detection of a therapeutic response, systemic treatment with an anti-firefly luciferase-targeting siRNA (siLuc) complexed with cationic nanoparticles was administered to mice with MLL-AF4 acute leukemia. The BLI signal showed a reduction after treatment with siLuc, compared to the control mice and the mice treated with a control siRNA.

\section{Materials and methods}

Cell lines. MLL-AF9 (THP-1 obtained from infant and MOLM-13 from adult patients) $(32,33)$ human acute monocytic leukemia cell lines were cultured in RPMI-1640 (Gibco BRL), containing $10 \%$ fetal bovine serum (FBS, EuroClone) and $1 \%$ penicillin/streptomycin ( $\mathrm{P} / \mathrm{S}$, Invitrogen). The MLL-AF4 (SEM) (9) human acute byphenotypic leukemia cell line was cultured in Dulbecco's modified Eagle's medium (DMEM, Invitrogen) containing $10 \% \mathrm{FBS}$ and $1 \% \mathrm{P} / \mathrm{S}$. The MLL-ENL (KOPN-8) (34) human B-cell precursor leukemia cell line was cultured in RPMI-1640 containing 20\% FBS and 1\% P/S. A retrovirus-packaging cell line for amphotropic retroviruses, Phoenix A, was maintained in DMEM containing 10\% FBS, and $1 \% \mathrm{P} / \mathrm{S}$. All cultures were maintained at $37^{\circ} \mathrm{C}$ and $5 \% \mathrm{CO}_{2}$.

Transduction of leukemia cells. The luciferase retroviral expression plasmid pMMP-Lucneo (kindly provided by Professor Andrew Kung, Harvard Medical School, Boston, MA) was transfected using Lipofectamine 2000 (Invitrogen) into Phoenix cells to generate the amphotropic retrovirus. Viral stock was collected 48 and 72 h post-transfection and was 
used to transduce leukemia cell lines by spinoculation in the presence of polybrene (hexadimethrine bromide, Sigma). Cells were incubated for $48 \mathrm{~h}$ in growth medium and then selected for 15 days with $1 \mathrm{mg} / \mathrm{ml}$ of G418 (Calbiochem). Positive cell clones where imaged using the light-tight chamber of a cooled CCD camera system (LB981, Berthold Technologies) and were named THP-1-luc, MOLM-13-luc, SEM-luc and KOPN-8-luc, respectively.

In vitro bioluminescence measurements. Cell clones with the highest light emission were selected with the luciferase assay reagent (Promega). A cell preparation $\left(10^{5}\right.$ cells in $\left.100 \mu 1 \mathrm{PBS}\right)$ was plated into a white 96 -well cell culture microplate and luminescence signals were measured following the addition of $100 \mu \mathrm{l}$ of luciferase assay reagent using a spectral scanning multimodal plate reader (Varioskan Flash, Thermo Scientific). Luminescence measurements were performed at room temperature $\left(25^{\circ} \mathrm{C}\right)$ and emission spectra were measured in the 560-670 $\mathrm{nm}$ range by collecting the light output every $2 \mathrm{~nm}$ for $1 \mathrm{sec}$. Serial dilutions of selected clones were plated in triplicate in a series starting from $10^{5}$ cells to assess the relationship between the viable cell count, determined by a trypan blue exclusion assay, and BL signals for each cell clone. After receiving $100 \mu \mathrm{l}$ of luciferase assay system substrate, the cells were imaged for $1 \mathrm{~min}$ with the same region of interest (ROI) used to measure luminescent signals of cells in each well using LB981 (Berthold Technologies). Linear regression analysis was used to determine the correlation between bioluminescence signal intensity and number of cells $\left(\mathrm{R}^{2}=0.98, \mathrm{p}<0.001, \mathrm{n}=3\right)$.

$R T-P C R$. RNA from each cell line was extracted using the RNeasy kit (Qiagen), following the manufacturer's protocol. Total RNA was quantified with the NanoDrop2000 spectrophotometer (Thermo Fisher Scientific). To generate first-strand cDNA, $1 \mu \mathrm{g}$ of total RNA was performed using Super Script II First Strand Synthesis Kit (Invitrogen) on thermocycler PTC 225 (MJ Research). RNA was incubated with random primers $(50 \mathrm{ng} / \mu \mathrm{l})$ and $\mathrm{dNTPs}(10 \mathrm{mM})$ for $5 \mathrm{~min}$ at $65^{\circ} \mathrm{C}$, then for $1 \mathrm{~min}$ at $4^{\circ} \mathrm{C}$. Then $15 \mathrm{mM} \mathrm{MgCl}$ containing buffer, DTT 0.1 M, and RNaseOUT ${ }^{\mathrm{TM}}$ Ribonuclease inhibitor $40 \mathrm{U} / \mu \mathrm{l}$ were added and the incubation was carried out for $2 \mathrm{~min}$ at $42^{\circ} \mathrm{C}$. Finally, Superscript ${ }^{\circledR} \mathrm{II}$ reverse transcriptase $50 \mathrm{U} / \mu 1$ was added with the following program: $50 \mathrm{~min}$ at $42^{\circ} \mathrm{C}, 15 \mathrm{~min}$ at $70^{\circ} \mathrm{C}$ and $30 \mathrm{~min}$ at $4^{\circ} \mathrm{C}$. The resulting cDNA was subjected to PCR with a mix of $0.2 \mu \mathrm{M}$ of each sense and antisense primers, $0.2 \mathrm{mM}$ dNTPs, $1.5 \mathrm{mM} \mathrm{MgCl}{ }_{2}$ containing buffer (Eppendorf) and 1.25 units Taq polymerase (Eppendorf) with the following parameters: $95^{\circ} \mathrm{C}$ for $1 \mathrm{~min} ; 39$ cycles of $94^{\circ} \mathrm{C}$ for $30 \mathrm{sec}, 60^{\circ} \mathrm{C}$ for $30 \mathrm{sec}$ and $72^{\circ} \mathrm{C}$ for $1 \mathrm{~min} ; 72^{\circ} \mathrm{C}$ for $7 \mathrm{~min}, 4^{\circ} \mathrm{C}$ for $10 \mathrm{~min}$. The sequences of the gene-specific primers were as follows: MLL-AF9 (THP-1) 5'-AGGACCGCCAAGAAAAGA-3' (sense); 5'-CCTGGTCT GGGATGGTGTGAA-3' (antisense), MLL-AF9 (MOLM-13) 5'-AGCACTCTCTCCAATGGC AATAGT-3' (sense); 5'-AAGGACCTTGTTGCCTGGTCTG-3' (antisense); MLL-AF4 5'-GCCCAAGTATCCCTGTA AAACA-3'(sense); 5'-TAGGGAAAGGAAACTTGGATGG-3' (antisense); MLL-ENL 5'-CACCAGAATCAGGTCC AGAGCA-3' (sense); 5'-TCCTTGGCTGTGGTTCTGGGAT-3' (antisense); human GAPDH 5'-CCAATCTGATTCCACCAT
GGC-3' (sense); 5'-CTTGATTTTGGAGGGATCTCGC-3' (antisense); murine GAPDH 5'-CCAAGGAGTAAGAAACC CTGGA-3' (sense); 5'-GGCCCCTCCTGTTATTATGG-3' (antisense). The amplification products were visualized on ethidium bromide-stained agarose gels.

Animal models. Female 6 week-old severe combined immunodeficient NOD/SCID mice were purchased from Charles River Laboratories. The mice were separated into different groups for each type of cell line and at least 10 mice for each cell line were used. Mice were inoculated intravenously with $5 \times 10^{6}$ MOLM-13-luc, SEM-luc or KOPN-8-luc cells, or $15 \times 10^{6}$ THP-1-luc cells. Mice were handled according to the international guidelines (the Helsinki Declaration) and the experiments were approved by the Bioethics Committee of Bologna University.

In vivo bioluminescence imaging. Based on previous experiments on the kinetics of the D-luciferin in vivo reaction (35), mice received an intraperitoneal (i.p.) injection of $150 \mathrm{mg} / \mathrm{kg}$ D-luciferin (Gold Biotechnology) and were placed in the LB981 (Berthold Technologies) under isoflurane anaesthesia (Ugobasile).Photographic and luminescent images were acquired starting 5 min after the D-luciferin injection, both in prone and supine position, for $3 \mathrm{~min}$. For all groups, leukemia progression was monitored every 7 days until mice were sacrificed due to the suffering from the extent of the leukemia dissemination. The Winlight software version 2.9 (Berthold Technologies) was used to analyze the BLI data. In vivo bioluminescence signals were calculated as the sum of both prone and supine acquisitions for each mouse after background subtraction (photon flux $(\mathrm{ph} / \mathrm{sec})$ from a total body ROI of $500 \mathrm{~mm}^{2}$ ). The BL curves for each xenograft leukemia model are reported as the means \pm SEM of the average of at least 10 mouse signals.

In vitro electroporation of small interfering RNA duplexes. Small interfering RNA (siRNA) against firefly luciferase gene (siLuc) and scrambled control (SCR) were purchased from Sigma: siLuc sense 5'-CUUACGCUGAGUACUUCGATT-3'; antisense 5'-UCGAAGUACUCAGCGUAAGTT-3'; SCR sense 5'-UAGCGACUAAACACAUCAAdTdT-3'; antisense 5'-UUG AUGUGUUUAGUCGCUAdTdT-3'.

Luciferase-expressing leukemia cell lines were electroporated using the Ingenio Electroporation Kit (Mirus-Bio) and the Nucleofector I Device (Lonza). A total of 1 million per sample was harvested by centrifugation at $1000 \mathrm{rpm}$ for $10 \mathrm{~min}$. Supernatant was removed and the cell pellet was resuspended in $105 \mu \mathrm{l}$ of the electroporation reagent. siLuc or SCR siRNA $(5 \mu \mathrm{g})$ were mixed with cells and subsequently transferred into electroporation cuvettes. MLL-AF4 acute biphenotypic leukemia SEM-luc cells were electroporated using the V-01 program. Following electroporation, $500 \mu \mathrm{l}$ of prewarmed complete medium was immediately added to the cells. Electroporated cells were seeded into 6-well culture plates containing $1.5 \mathrm{ml}$ of prewarmed medium per well. Four hours after electroporation, $2 \mathrm{ml}$ of prewarmed complete medium per well was added, and $100 \mu \mathrm{l}$ of cells in triplicate were plated in 96-well culture plates for both luciferase and viability cell assay at 24 and $48 \mathrm{~h}$ post-transfection. Luciferase assay was performed as previously described. 
In vivo siRNA delivery. In vivo-jetPEI ${ }^{\mathrm{TM}}$ (optimized cationic linear PEI-based transfection reagent for in vivo experiments) was obtained from Polyplus-transfection (Illkirch). Fifty micrograms of siLuc or SCR siRNA as negative control were diluted in $100 \mu \mathrm{l}$ of $5 \%$ glucose solution. In vivo-jetPEI was used at N/P ratio of 10; in vivo-jetPEI was diluted in $100 \mu \mathrm{l}$ of $5 \%$ glucose solution and mixed by vortexing for $10 \mathrm{sec}$. The in vivo-jetPEI diluted solution was added to the nucleic acid solution, mixed by vortexing for $10 \mathrm{sec}$ and incubated for at least $15 \mathrm{~min}$ at RT before injecting into the mice. Treatments were performed on MLL-AF4 acute biphenotypic leukemia SEM-luc mice, starting 1 week after inoculation, and repeated every $48 \mathrm{~h}$ by tail vein injection. Bioluminescence analysis was performed every week as described above.

Organ collection. Blood samples were collected from the orbital sinus with micro-haematocrit capillary tubes (Brand). A blood smear for each sample was performed and stained with the May-Grünwald-Giemsa stain. Briefly, few microliters of blood were used for blood smears. The blood slides were stained for $3 \mathrm{~min}$ in the May-Grünwald solution, washed with distilled water, stained for $7 \mathrm{~min}$ in the Giemsa solution, then washed again with distilled water, and finally let dry. Slides were observed at the optic microscope to evaluate the leukemic infiltration. Bone marrow, spleen and liver of dead mice were collected for molecular analysis. RNA from mouse tissues were extracted with Trizol Reagent (Invitrogen), following the manufacturer's protocol. RT-PCR on mice RNA was performed as previously described. The spleen of leukemic and control mice were compared and photographed to evaluate the infiltration of leukemia cells.

\section{Results}

In vitro BLI imaging. We confirmed the linear relationship between cell number and bioluminescence signals by the luciferase assay, that appeared highly proportional to cell number in a linear range (Fig. 1A and B). The correlation coefficient was $>0.99$ for all cell clones. At the same plated concentration, the highest bioluminescence signal was from THP-1-luc cells, followed by SEM-luc; the KOPN-8-luc and MOLM-13-luc cell lines showed the lowest BL intensity. The same trend of bioluminescent signal was noted in the emission spectra of intact cell clones (Fig. 1C). At an emission maximum of $562 \mathrm{~nm}$, $10^{5}$ cells of THP-1-luc and SEM-luc emitted at about $2 \times 10^{3}$ RLU, MOLM-13-luc and KOPN-8-luc at slightly $<1 \times 10^{3}$. The luciferase expression was not found to have any significant effects on the expression of MLL fusion genes, proteins or proliferation rates of the four cell lines.

MLL-related acute leukemia progression evaluated by BLI. All animals developed MLL-related acute leukemias as detected by in vivo BLI. The four MLL-related acute leukemia mouse models showed different progressions, due to the inter-variability among the MLL fusion genes, and the intravariability between the infant and adult MLL-AF9 leukemias (Fig. 2A and B). In three of the four models, MLL-AF4 acute biphenotypic leukemia, MLL-ENL ALL and adult MLL-AF9 AML, leukemia developed in the bone marrow and progressed to a more disseminated disease resembling human leukemia, whereas in the infant MLL-AF9 AML model leukemia development and progression was restricted to the bone marrow. The adult MLL-AF9 AML model presented a latency time of three weeks and an end point of four weeks, which was the shortest time among the four models. It also showed diffuse metastasis in the subcutaneous and osseous planes, with sporadic infiltration of lymphatic structures as lymph nodes. The infant MLL-AF9 AML model had the slowest latency time; it developed leukemia at the third week, and showed the lowest BL signal among the four models. Furthermore, the infant MLL-AF9 AML model did not show dissemination of human leukemia cells into the blood system. The MLL-ENL ALL model revealed the shortest latency time among the four models, and the BL signal was already evident at the second week after cell inoculation. The end point of the MLL-ENL ALL mice was at the fifth week, the BL signal of MLL-ENL ALL progression was also the highest among the four models and spread in the whole body, due to dissemination in various organs. The MLL-AF4 acute biphenotypic leukemia model showed a latency time of two weeks and the end point was six weeks. The MLL-AF4 acute biphenotypic leukemia mice also developed alopecia on the head and back due to the infiltration of the leukemic cells under the skin.

Clinical findings. To confirm the in vivo BLI results, clinical macroscopic observations on organs were performed. Mouse spleens were compared to evaluate the splenomegaly due to infiltration of human leukemia cells (Fig. 3A). The MLL-AF4 acute biphenotypic leukemia model showed a strong infiltration into the spleen with evident splenomegaly. Peripheral blood smears were performed at the end point of mice to evaluate the blood infiltration of human leukemia cells and were compared to peripheral blood smears of control mice (Fig. 3B) and to cell lines alone (Fig. 3C). MLL-AF4 biphenotypic leukemia, MLL-ENL ALL and adult MLL-AF9 revealed dissemination of leukemia cell lines into the blood system, except for the infant MLL-AF9 AML cells, as we had expected.

Tissue molecular analysis. Bone marrow, spleen and liver from mice were analyzed by RT-PCR and were found differently positive for MLL fusion gene expression (Fig. 4). Human GAPDH housekeeping mRNA was found negative in samples from control mice and positive in samples from leukemic mice, excepted for the spleen and liver of mice inoculated with infant MLL-AF9 AML cells, confirming the BL results; murine GAPDH (mGAPDH) housekeeping mRNA was found positive in all mice samples and negative in human leukemic cell lines, confirming the specificity of the RNA extraction from mice. The MLL-AF9 mRNA was found positive only in the bone marrow sample from adult MLL-AF9 AML mice, showing the two previously described mRNA splicing isoforms of MLL-AF9 in MOLM-13 cells (36). The MLL-AF4 mRNA was found positive in all the tissues from MLL-AF4 acute biphenotypic leukemia mice. The MLL-ENL mRNA was found clearly positive in the bone marrow and less clear but present in the liver of the MLL-ENL ALL mice.

In vitro transfection of siRNA. We transiently transfected the MLL-AF4 acute biphenotypic leukemia SEM-luc cell line 
A

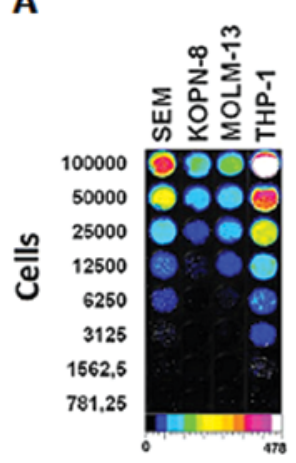

B

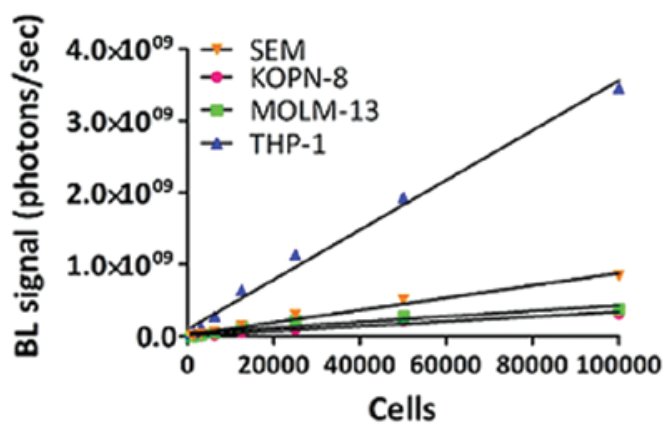

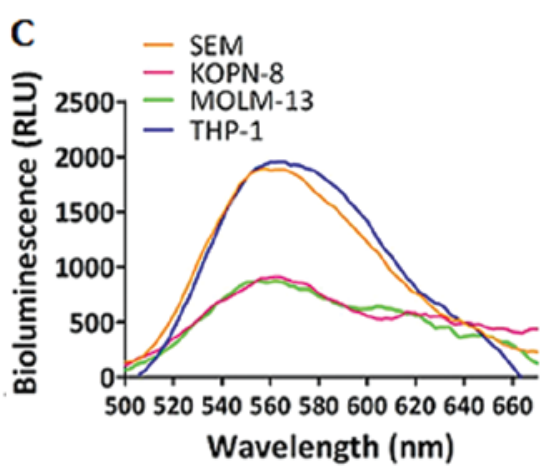

Figure 1. (A) Pseudocolor image of flux values in photons/sec for serial dilutions of THP-1-luc, MOLM-13-luc, SEM-luc, KOPN-8-luc cell lines. (B) Correlation between cell numbers and luminescent signals $\left(R^{2}=0.98, p<0.001, n=3\right)$. (C) Emission spectra in the $560-670 \mathrm{~nm}$ range.

A

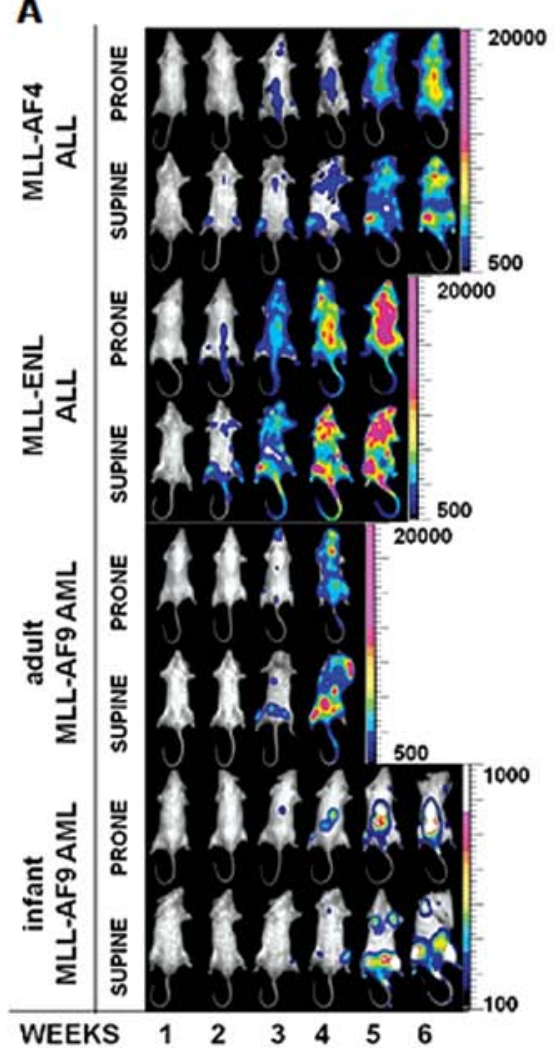

B

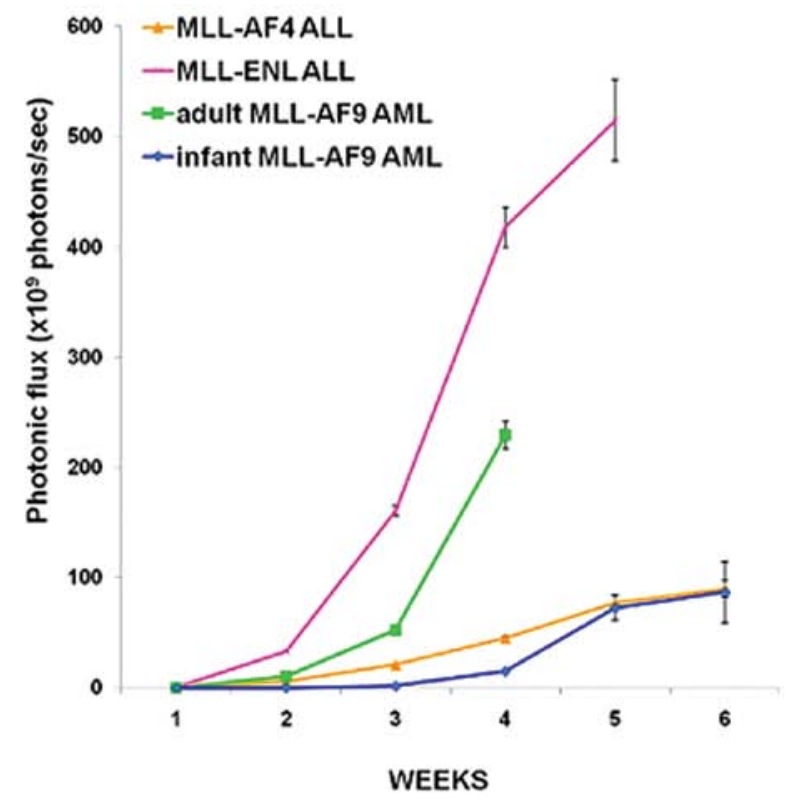

Figure 2. (A) In vivo BLI monitored every 7 days in NOD/SCID mice inoculated with MOLM-13-luc, SEM-luc, KOPN-8-luc, THP-1-luc cells. (B) BL signal reported as mean of photon flux \pm SEM.

with a siRNA against luciferase mRNA (siLuc) and with a scrambled siRNA (siSCR). The luminescence analysis showed in vitro reduction of luminescence signal in the samples treated with siLuc compared to the control samples or the samples treated with siSCR, at 24 and $48 \mathrm{~h}$ post-transfection (Fig. 5).

In vivo siRNA delivery. We treated MLL-AF4 acute biphenotypic leukemia (SEM-luc) mice with siLuc and we monitored the BL signal during the treatment. We compared the BL signal in treated mice with siSCR-treated or untreated control mice (Fig. 6). We observed a statistically significant difference between BL signals in siLuc and siSCR-treated mice from the fourth week of treatment to the end point, confirming

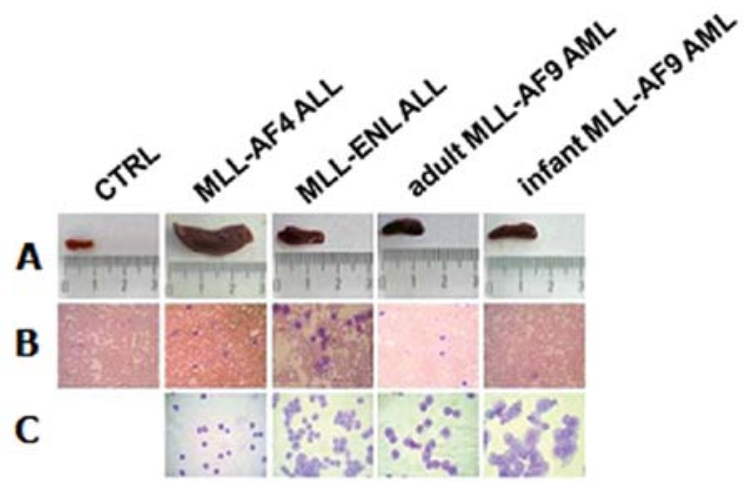

Figure 3. (A) Spleens of leukemic and control mice. (B) Peripheral blood smears stained with May-Grünwald-Giemsa stain. (C) Control cells. 


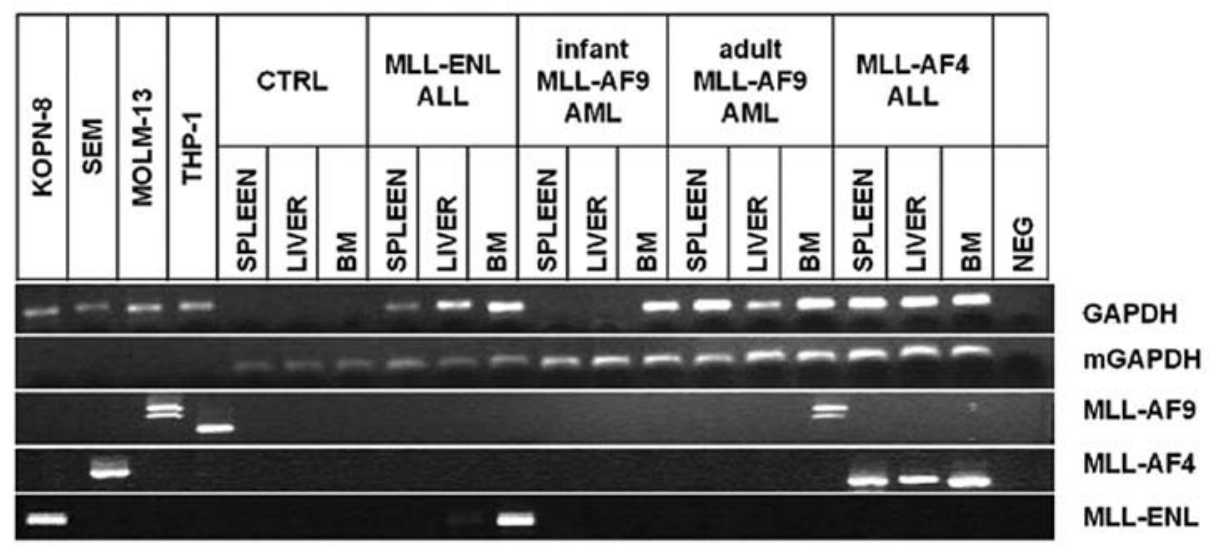

Figure 4. Qualitative MLL-fusion gene expression of MLL-related cell lines and in the bone marrow, spleen and liver of control and human leukemia cellinoculated mice.

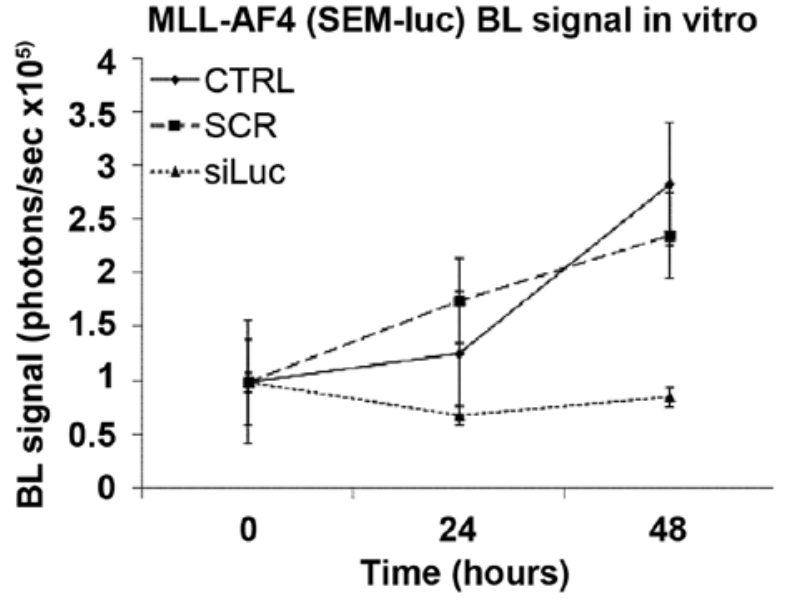

Figure 5. In vitro monitoring of luciferase expression inhibition by siLuc in MLL-AF4-positive SEM-luc cells.

the specificity of the luciferase gene downregulation and the validity of the mouse model (Fig. 6A and B).

\section{Discussion}

Our bioluminescent mouse models of the most frequent and high risk MLL-related acute leukemias provide a non-invasive, sensitive, rapid and affordable method to study the different behaviors of leukemia progression. The high sensitivity of in vivo BLI enables the early detection of tumors, either superficially or in deep tissues such as the bone marrow, preceding the appearance of symptoms (25).

Moreover, our MLL-related acute leukemia mouse models show the different course of infant and adult human MLL-AF9 AML, and the rapid aggressiveness of human MLL-ENL ALL and MLL-AF4 acute biphenotypic leukemia. Among the four models, three, MLL-AF4 acute biphenotypic leukemia, MLL-ENL ALL and adult MLL-AF9 AML, developed leukemias in the bone marrow and progressed to a more disseminated disease, and the results were confirmed by in vivo BLI, clinical findings and RT-PCR molecular analysis on tissues. Leukemia development and progression was
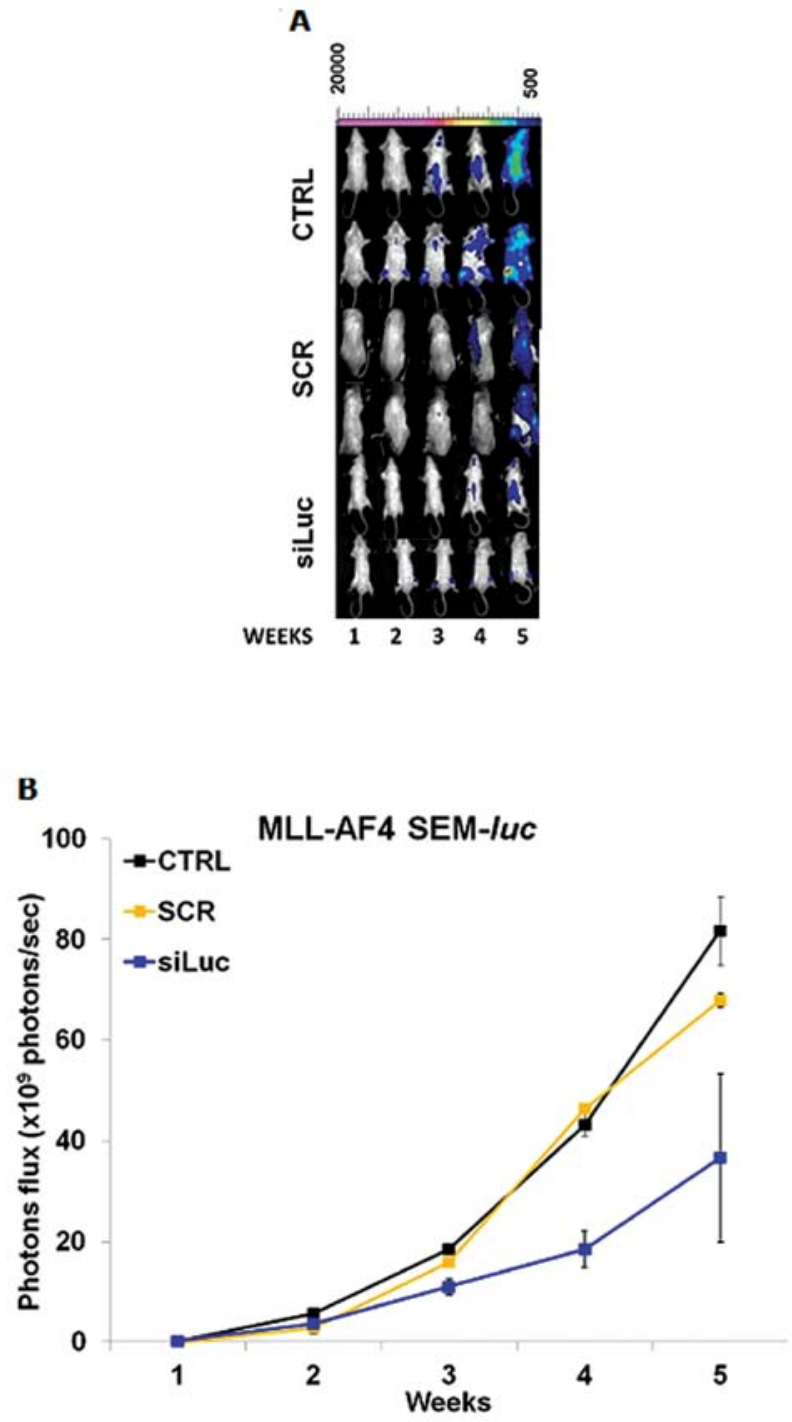

Figure 6. (A) In vivo monitoring of luciferase expression inhibition by siLucnanoparticle complex in mice with MLL-AF4 positive SEM-luc leukemia. (B) BL signal reported as mean of photon flux \pm SEM. 
restricted to the bone marrow only in the infant MLL-AF9 AML model; this behavior is possibly due to the lower infiltration trend of infant MLL-AF9 AML. The two MLL-AF9 AML models showed a different course, probably due to their different origin: the THP-1 cell line was established from the peripheral blood of a 1-year-old boy with subtype M5a acute monocytic leukemia (32), whereas the MOLM-13 cell line was established from the peripheral blood of a 20 -year-old male patient at relapse of acute monocytic leukemia, subtype M5a, which had evolved from myelodysplastic syndrome (33). In vivo BLI allowed us to distinguish the different courses of leukemia growth in infant and adult MLL-AF9 AML models, and enabled the study of the mechanism of action of the same MLL-AF9 oncogene in different contexts, such as in infant or adult patients.

The parallelism between the results of in vivo BLI and the clinical course of MLL-related acute leukemias supports our proposed models as suitable tools for the study of MLL-leukemias.

Moreover, our in vivo BLI models may be complementary to transgenic mouse models of MLL-related acute leukemias due to their capability to reveal different details of disease progression and could be helpful for the leukemia research community to elucidate the trafficking in vivo of the different MLL-related acute leukemias.

The accurate definition of the leukemic evolution from the early beginning to the final stages in these MLL-related acute leukemia mouse models represents optimal characteristics for the definition of preclinical treatment schedules in drug development studies for MLL-related acute leukemias.

To confirm this hypothesis, we treated the MLL-AF4 acute biphenotypic leukemia bioluminescent mice with a specific anti-firefly luciferase siRNA, complexed with polyethylenimine-based nanoparticles to protect them from serum nuclease degradation and to deliver the siRNA into the cells. We chose the MLL-AF4 acute biphenotypic leukemia mouse model due to the poor prognosis of MLL-AF4 acute biphenotypic leukemias in pediatric patients and due to the widest window of latency and progression in mice between all our leukemia mouse models. We monitored the in vivo siRNAmediated downregulation of luciferase activity by in vivo BLI. We observed a significant lower BL signal in treated mice compared to untreated control mice and to siSCR-treated mice.

Finally, we propose our MLL-related acute leukemia bioluminescent mouse model for in vivo siRNA therapeutic treatments as a powerful tool to validate the efficacy of muchneeded new therapies for this aggressive group of leukemias.

\section{Acknowledgements}

This work was supported by the CARISBO Foundation, the del Monte Foundation of Bologna and Ravenna, AIRC and AGEOP.

\section{References}

1. Krivtsov AV and Armstrong SA: MLL translocations, histone modifications and leukaemia stem-cell development. Nat Rev Cancer 7: 823-833, 2007.

2. Bach C and Slany RK: Molecular pathology of mixed-lineage leukemia. Future Oncol 5: 1271-1281, 2009.
3. Thirman MJ, Gill HJ, Burnett RC, et al: Rearrangement of the MLL gene in acute lymphoblastic and acute myeloid leukemias with 11q23 chromosomal translocations. N Engl J Med 329: 909-914, 1993

4. Meyer C, Kowarz E, Hofmann J, et al: New insights to the MLL recombinome of acute leukemias. Leukemia 23: 1490-1499, 2009.

5. Pui CH, Chessells JM, Camitta B, et al: Clinical heterogeneity in childhood acute lymphoblastic leukemia with 11q23 rearrangements. Leukemia 17: 700-706, 2003.

6. Pui $\mathrm{CH}$, Schrappe M, Ribeiro RC and Niemeyer CM: Childhood and adolescent lymphoid and myeloid leukemia. Hematology Am Soc Hematol Educ Program, pp118-145, 2004.

7. Hess JL: MLL: a histone methyltransferase disrupted in leukemia. Trends Mol Med 10: 500-507, 2004.

8. Pizzo PA and Poplack DG: Principles and Practice of Pediatric Oncology. 5th Edition. Lippincott Williams \& Wilkins, Philadelphia, PA, 2006.

9. Greil J, Gramatzki M, Burger R, et al: The acute lymphoblastic leukaemia cell line SEM with $\mathrm{t}(4 ; 11)$ chromosomal rearrangement is biphenotypic and responsive to interleukin-7. Br J Haematol 86: 275-283, 1994.

10. Balgobind BV, Raimondi SC, Harbott J, et al: Novel prognostic subgroups in childhood 11q23/MLL-rearranged acute myeloid leukemia: results of an international retrospective study. Blood 114: 2489-2496, 2009.

11. Frascella E, Rondelli R, Pigazzi M, et al: Clinical features of childhood acute myeloid leukaemia with specific gene rearrangements. Leukemia 18: 1427-1429, 2004

12. Dothager RS, Flentie K, Moss B, Pan MH, Kesarwala A and Piwnica-Worms D: Advances in bioluminescence imaging of live animal models. Curr Opin Biotechnol 20: 45-53, 2009.

13. Luker GD and Luker KE: Optical imaging: current applications and future directions. J Nucl Med 49: 1-4, 2008.

14. Klerk CP, Overmeer RM, Niers TM, et al: Validity of bioluminescence measurements for noninvasive in vivo imaging of tumor load in small animals. Biotechniques 43: 7-13, 2007.

15. Greer LF and Szalay AA: Imaging of light emission from the expression of luciferases in living cells and organisms: a review. Luminescence 17: 43-74, 2002.

16. Söling A and Rainov NG: Bioluminescence imaging in vivoapplication to cancer research. Expert Opin Biol Ther 3: 1163-1172, 2003. Erratum in: Expert Opin Biol Ther 3: 1315, 2003.

17. Negrin RS and Contag $\mathrm{CH}$ : In vivo imaging using bioluminescence: a tool for probing graft-versus-host disease. Nat Rev Immunol 6: 484-490, 2006.

18. Inoue Y, Tojo A, Sekine R, et al: In vitro validation of bioluminescent monitoring of disease progression and therapeutic response in leukaemia model animals. Eur J Nucl Med Mol Imaging 33: 557-565, 2006.

19. Inoue $\mathrm{Y}$, Izawa $\mathrm{K}$, Tojo A, et al: Monitoring of disease progression by bioluminescence imaging and magnetic resonance imaging in an animal model of hematologic malignancy. Exp Hematol 35: 407-415, 2007.

20. Inoue Y, Izawa K, Kiryu S, Kobayashi S, Tojo A and Ohtomo K: Bioluminescent evaluation of the therapeutic effects of total body irradiation in a murine hematological malignancy model. Exp Hematol 36: 1634-1641, 2008.

21. Armstrong SA, Kung AL, Mabon ME, et al: Inhibition of FLT3 in MLL: Validation of a therapeutic target identified by gene expression based classification. Cancer Cell 3: 173-183, 2003.

22. Edinger M, Cao YA, Verneris MR, Bachmann $\mathrm{MH}$, Contag $\mathrm{CH}$ and Negrin RS: Revealing lymphoma growth and the efficacy of immune cell therapies using in vivo bioluminescence imaging. Blood 15: 640-648, 2003.

23. Maes W, Deroose C, Reumers V, et al: In vivo bioluminescence imaging in an experimental mouse model for dendritic cell based immunotherapy against malignant glioma. J Neurooncol 91: 127-139, 2009.

24. Inoue Y, Kiryu S, Izawa K, Watanabe M, Tojo A and Ohtomo K: Comparison of subcutaneous and intraperitoneal injection of D-luciferin for in vivo bioluminescence imaging. Eur J Nucl Med Mol Imaging 36: 771-779, 2009.

25. Wetterwald A, van der Pluijm G, Que I, et al: Optical imaging of cancer metastasis to bone marrow: a mouse model of minimal residual disease. Am J Pathol 160: 1143-1153, 2002.

26. Aronin N: Target selectivity in mRNA silencing. Gene Ther 13: 509-516, 2006.

27. Meister G and Tuschl T: Mechanisms of gene silencing by double-stranded RNA. Nature 431: 343-349, 2004. 
28. Tuschl T: RNA interference and small interfering RNAs. Chembiochem 2: 239-245, 2001.

29. Sledz CA and Williams BR: RNA interference in biology and disease. Blood 106: 787-794, 2005.

30. Cejka D, Losert D and Wacheck V: Short interfering RNA (siRNA): tool or therapeutic? Clin Sci 110: 47-58, 2006.

31. Behlke MA: Progress towards in vivo use of siRNAs. Mol Ther 13: 644-670, 2006.

32. Tsuchiya S, Yamabe M, Yamaguchi Y, Kobayashi Y, Konno T and Tada K: Establishment and characterization of a human acute monocytic leukemia cell line (THP-1). Int J Cancer 26: 171-176, 1980.

33. Matsuo Y, MacLeod RA, Uphoff CC, et al: Two acute monocytic leukemia (AML-M5a) cell lines (MOLM-13 and MOLM-14) with interclonal phenotypic heterogeneity showing MLL-AF9 fusion resulting from an occult chromosome insertion, ins $(11 ; 9)$ (q23;p22p23). Leukemia 11: 1469-1477, 1997.
34. Matsuo Y and Drexler HG: Establishment and characterization of human B cell precursor-leukemia cell lines. Leuk Res 22: 567-579, 1998.

35. Mezzanotte L, Fazzina R, Michelini E, et al: In vivo bioluminescence imaging of murine xenograft cancer models with a red-shifted thermostable luciferase. Mol Imaging Biol 12: 406-414, 2010.

36. Montemurro L, Tonelli R, Fazzina R, Martino V, Marino F and Pession A: Identification of two MLL-MLLT3 (alias MLL-AF9) chimeric transcripts in the MOLM-13 cell line. Cancer Genet Cytogenet 154: 96-97, 2004. 